\title{
Anesthetic management of a patient with airway stenosis due to a giant goiter and severe pulmonary hypertension: A case report
}

\author{
Akihisa Taguchi ${ }^{1}$, Akiko Hirotsu ${ }^{1}$, Misaki Saito Sato ${ }^{1}$, and Toshiyuki Mizota ${ }^{1}$ \\ ${ }^{1}$ Kyoto University Hospital
}

November 6, 2021

\begin{abstract}
Anesthetic management of patients with severe pulmonary hypertension combined with a difficult airway is challenging. The present case suggests that the use of venoarterial extracorporeal membrane oxygenation is useful for safe anesthetic management.
\end{abstract}

\section{Introduction}

Anesthetic management of patients with pulmonary hypertension $(\mathrm{PH})$ requires minimizing stress on the right heart. However, management becomes even more challenging when it is complicated by a difficult airway. It has been reported that venovenous extracorporeal membrane oxygenation (VV-ECMO) can be used for safe anesthetic management in patients with a difficult airway. ${ }^{1}$ However, the use of VV-ECMO can be problematic in cases complicated by $\mathrm{PH}$ because circulatory failure could occur after the induction of anesthesia. To our knowledge, there are no reports on the anesthetic management of patients with severe $\mathrm{PH}$ combined with a difficult airway. In this paper, we report a case of anesthetic management using femoral venoarterial ECMO (VA-ECMO) in a patient with severe $\mathrm{PH}$ who was expected to have a difficult airway because of a giant goiter.

\section{Case Presentation}

The patient was a 42-year-old female with a history of Graves' disease. At the age of 33, she was diagnosed with heritable pulmonary arterial hypertension and started medical treatment with a continuous intravenous infusion of prostacyclin. However, she was unresponsive to the medical treatment (World Health Organization functional class 3) and was enrolled as a recipient of brain dead-donor lung transplantation. Neck computed tomography at the time of transplant registration showed that the thyroid gland was compressing her trachea and that the narrowest part of the trachea was approximately $3.6 \mathrm{~mm}$ (Fig. 1). She was therefore scheduled for total thyroidectomy and tracheostomy. Right heart catheterization revealed precapillary PH with an elevated mean pulmonary artery pressure of $61 \mathrm{mmHg}$ and an elevated pulmonary vascular resistance of 11.7 Wood units. Transthoracic echocardiography (TTE) showed right ventricle enlargement that was compressing the left ventricle throughout the cardiac cycle. Because a difficult airway due to massive goiter and circulatory failure caused by severe $\mathrm{PH}$ was expected, we planned to induce anesthesia under VA-ECMO standby.

During the night on the day of admission, the patient suddenly developed dyspnea, and her level of consciousness decreased. We urgently attempted awake tracheal intubation using a video laryngoscope and a flexible intubation scope. We were able to identify a small portion of her vocal cords, but all of the attempts were unsuccessful because of her cough reflex; alternating between bag-valve-mask ventilation and sputum suctioning improved oxygenation and enabled the patient to communicate. Because of concerns about the recurrence of ventilation failure, emergency thyroidectomy was performed. 
As planned, anesthesia was induced after sheaths were placed in the femoral artery and vein. After induction of anesthesia, face-mask ventilation using an oral airway and intubation using a video laryngoscope or flexible intubation scope were difficult to perform, so femoral VA-ECMO was introduced immediately. Percutaneous oxygen saturation was maintained above $90 \%$ until the induction of VA-ECMO. After the initiation of VA-ECMO, we continued face-mask ventilation to prevent differential hypoxia, and tidal volume was approximately $100-150 \mathrm{~mL}$ When percutaneous oxygen saturation in the right hand decreased to $55 \%$ during the intubation attempt, we responded by increasing the ECMO flow. Using a combination of video laryngoscope and flexible intubation scope, we were able to intubate the patient 70 minutes after VA-ECMO induction. The surgery was completed with VA-ECMO support without any problems. However, we were not able to wean the patient from VA-ECMO immediately after surgery because of a diffuse left ventricular wall-motion abnormality. In the intensive care unit, wall-motion gradually improved with the use of intravenous epinephrine and dopamine, and the patient was able to be weaned from VA-ECMO support on the day after surgery. With the improvement in cardiac wall motion, epinephrine and dopamine were discontinued on postoperative days 4 and 12, respectively. The patient showed no neurologic abnormalities.

\section{Discussion}

$\mathrm{PH}$ is an independent risk factor for perioperative complications, and the reported complication rates for noncardiac surgery are as high as $14 \%-42 \% .^{2}$ For this reason, we initially planned to perform lung transplantation before thyroid surgery. However, the thyroid gland enlargement progressed quickly, and we expected airway management to be problematic during lung transplantation. Therefore, we decided to perform thyroid surgery first. Considering that the patient developed dyspnea due to airway obstruction on the day of admission, we believe that decision was appropriate.

Practice guidelines for the management of a difficult airway recommend tracheostomy or awake intubation before the induction of general anesthesia. ${ }^{3}$ However, because the distance from skin to the trachea is greater in patients with a large goiter, tracheostomy before the induction of general anesthesia would have been difficult in our case. Awake intubation was also difficult because the patient could not tolerate it, and we could barely visualize her vocal cords. In such situations, VV-ECMO is often chosen to support the induction of anesthesia. ${ }^{1}$ However, several risk factors for perioperative complications in patients with $\mathrm{PH}$ undergoing noncardiac surgery have been identified (Table 1). ${ }^{2,4}$ VA-ECMO is preferred for circulatory support in diseases that can cause right ventricular failure, such as $\mathrm{PH}$ and pulmonary embolism. ${ }^{5}$ Therefore, we selected VA-ECMO rather than VV-ECMO for our patient because she was at high risk for perioperative complications such as right heart failure. With the induction of VA-ECMO, we were able to reduce right ventricular preload and afterload, thus stabilizing the patient's right heart performance.

Postoperative TTE showed a diffuse left ventricular wall-motion abnormality, suggesting acute left heart failure. The causes may have been temporary myocardial ischemia due to differential hypoxia or increased left ventricular afterload due to retrograde ECMO flow. Differential hypoxia, which is particularly common in femoral VA-ECMO, could result in insufficient oxygen supply to the brain and the heart. To manage differential hypoxia, we increased ECMO flow to reduce left ventricular stroke volume and continued facemask ventilation until intubation was successful. With increased ECMO flow, pulmonary blood flow was reduced, and a smaller minute volume was sufficient. We considered these procedures to be appropriate based on percutaneous oxygen saturation in the right hand. ${ }^{6}$ However, the higher the ECMO flow, the greater the left ventricular afterload, increasing the risk of left heart failure. Some studies have reported using venoarterial-venous ECMO (i.e., a hybrid ECMO configuration that combines the cardiac support of VA-ECMO with the gas exchange support of VV-ECMO) in patients with hemodynamic instability and marked respiratory failure. ${ }^{7,8}$ Venoarterial-venous ECMO is certainly an interesting method to prevent differential hypoxia and excessive left ventricular afterload, but this configuration adds greater complexity to management and needs more time to be induced. Lindfors et al. reported that positioning the tip of the venous cannula of femoral VA-ECMO near the superior vena cava improved oxygen saturation in the ascending aorta from $60 \%$ to $80 \% .^{9}$ This method could have been more easily applied than venoarterialvenous ECMO and might have been useful in our case. 
Patients with severe $\mathrm{PH}$ and a difficult airway often require respiratory and circulatory support, making their management very challenging for anesthesiologists. In our case, femoral VA-ECMO was useful for safe anesthetic management.

\section{Author contribution}

AT: helped manage the anesthetic care and write the article. AH: helped manage the perioperative care, critically revise and approve the manuscript. MSS: helped manage the anesthetic care, critically revise and approve the manuscript. TM: helped manage the perioperative care, critically revise and approve the manuscript.

\section{References}

1. Malpas G, Hung O, Gilchrist A, et al. The use of extracorporeal membrane oxygenation in the anticipated difficult airway: A case report and systematic review. Can J Anaesth 2018;65:685-697.

2. Pilkington SA, Taboada D, Martinez G. Pulmonary hypertension and its management in patients undergoing non-cardiac surgery. Anaesthesia 2015;70:56-70.

3. Apfelbaum JL, Hagberg CA, Caplan RA, et al. Practice guidelines for management of the difficult airway: An updated report by the American society of anesthesiologists task force on management of the difficult airway. Anesthesiology 2013;118:251-270.

4. Minai OA, Yared JP, Kaw R, Subramaniam K, Hill NS. Perioperative risk and management in patients with pulmonary hypertension. Chest 2013;144:329-340.

5. Machuca TN, de Perrot M. Mechanical support for the failing Right Ventricle in Patients With Precapillary Pulmonary Hypertension. Circulation. 2015;132:526-536

6. Antoniucci ME, De Paulis S, Bevilacqua F, et al. Unconventional cannulation strategy in peripheral extracorporeal membrane oxygenation to achieve central perfusion and prevent differential hypoxia. J Cardiothorac Vasc Anesth. 2019;33:1367-9

7. Ius F, Sommer W, Tudorache I, et al. Veno-veno-arterial extracorporeal membrane oxygenation for respiratory failure with severe haemodynamic impairment: Technique and early outcomes. Interact Cardiovasc Thorac Surg 2015;20:761-767.

8. Choi JH, Kim SW, Kim YU, et al. Application of veno-arterial-venous extracorporeal membrane oxygenation in differential hypoxia. Multidiscip Respir Med 2014;9:55.

9. Lindfors M, Frenckner B, Sartipy U, Bjällmark A, Broomé M. Venous cannula positioning in arterial deoxygenation during veno-arterial extracorporeal membrane oxygenation-a simulation study and case report. Artif Organs 2017;41:75-81.

\section{Figure captions}

Fig 1. Neck computed tomography before surgery. The narrowest part of the trachea was approximately $3.6 \mathrm{~mm}$, and the thyroid gland extended from the base of the tongue to the supraclavicular region.

Table 1. Identified risk factors for perioperative complications in patients with preoperative $\mathrm{PH}$ undergoing noncardiac surgery.

\section{Patient factors}

ASA physical status $>2$

NYHA/WHO functional class [?]2

Underlying coronary artery disease

Higher mean pulmonary artery pressure

Co-morbidities Pulmonary embolism Obstructive sleep apnea Chronic renal insufficiency Untreated pulmonary hypertensior RVH with severe systolic dysfunction

RA dilation

Abbreviations: ASA, American Society of Anesthesiologists; NYHA, New York Heart Association; WHO, 
World Health Organization; RVH, right ventricular hypertrophy; RA, right atrial

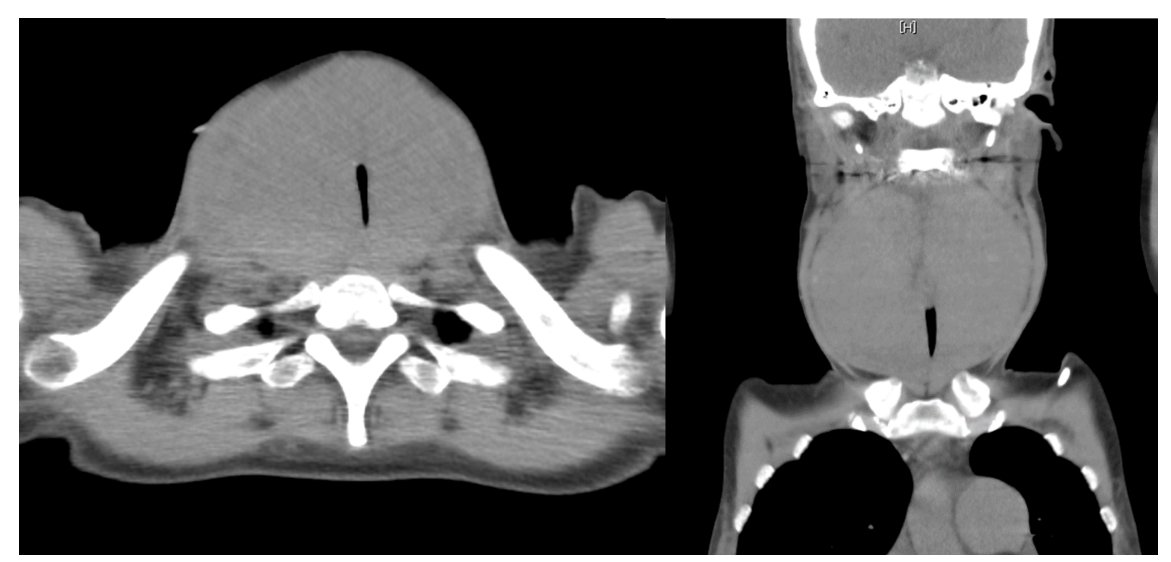

